\title{
Tratamiento quirúrgico del cáncer gástrico
}

\section{Surgical treatment of gastric cancer}

\author{
Eloy Ruiz
}

\section{Resumen}

Para los pacientes con cáncer gástrico localizado (T1-4, N0-3, M0), la resección oncológica del tumor es el único tratamiento potencialmente curativo. La resección oncológica debe alcanzar necesariamente dos objetivos: a) Extirpación del tumor con márgenes libres y b) Resección de ganglios linfáticos regionales comprometidos. Debido a la heterogeneidad del cáncer gástrico, el tipo de resección gástrica (mucosectomía, gastrectomía o gastrectomía extendida) y linfadenectomía (D2, D1, D2) depende de la profundidad de invasión de la pared gástrica y la posibilidad de metástasis en los ganglios linfáticos. Por lo tanto, utilizando todos los métodos de diagnóstico, el TNM clínico (cTNM) debe establecerse en el preoperatorio para realizar una resección orientada por estadios.

Palabras clave: Cáncer gástrico, gastrectomía, linfadenectomia. .

\section{Abstract}

For patients with Localized Gastric Cancer (T1-4, N0-3, M0) the oncologic resection of the tumor is the only potentially curative treatment. The oncologic resection is necessary to achieve two objectives: a) Extirpation of the tumor with free margins and b) Resection of regional lymph nodes. Due to the heterogeneity of gastric cancer, the type of gastric resection (mucosectomy, gastrectomy or extended gastrectomy) and lymphadenectomy (D2, D1, D2) depends of the depth of invasion of the gastric wall and the possibility of lymph node metastases. Therefore, using all methods of diagnosis, the clinical TNM (cTNM) has to be established in the pre-operative period in order to perform a stage oriented resection.

Keywords: Gastric cancer, gastrectomy, lymphadenectomy.

\section{Introducción}

El Adenocarcinoma de estómago o cáncer gástrico se origina en las células epiteliales de la mucosa del estómago, si el paciente no recibe tratamiento y desarrolla la historia natural de esta neoplasia, transcurren de 16.5 a 33 años entre la aparición de las primeras células neoplásicas y la muerte del paciente. - En los primeros años el tumor incrementa sus dimensiones muy lentamente y permanece localizado en la mucosa, pero luego la velocidad de duplicación se acelera y el tumor crece en todas las direcciones e invade en profundidad las diferentes capas de la pared gástrica.

De las múltiples clasificaciones utilizadas para describir la invasión en profundidad de la pared la más usada es la propuesta por la AJCC-UICC ${ }^{(1)}$ (Tabla 1).

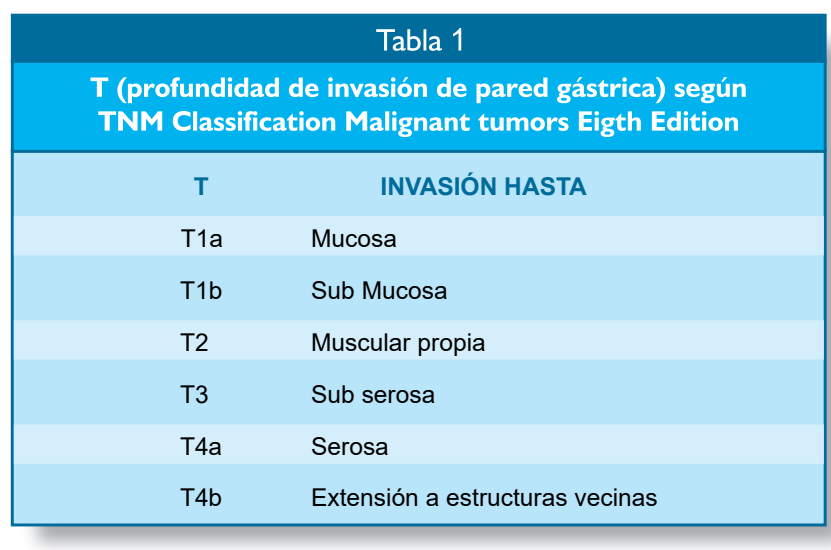

El tumor también invade capilares linfáticos y sanguíneos dando origen a metástasis ganglionar y metástasis a distancia respectivamente.

${ }^{\prime}$ Jefe Departamento de Cirugía en el Instituto de Enfermedades Neoplásicas (INEN), Lima - Perú. orcid.org/0000-0001-5561-0752. 
La metástasis en los ganglios que rodean el estómago (Grupos 1-12) se considera como metástasis ganglionar regional $(\mathrm{N})$, pero la metástasis en ganglios para aórticos, supra clavicular y umbilical se consideran metástasis a distancia (M1).

La metástasis a distancia de origen hematógeno (por ejemplo en hígado, pulmón, hueso etc.) y las producidas por implantes en peritoneo (Carcinomatosis peritoneal) se catalogan como M1.

Los pacientes con metástasis a distancia tienen un Cáncer Gástrico Diseminado (CGD) y no tienen posibilidad de curación, los múltiples tratamientos empleados (Quimioterapia, Radioterapia, resecciones o derivaciones paliativas) no incrementan significativamente la sobrevida y el paciente inexorablemente fallece por progresión de la enfermedad. Lamentablemente al momento del diagnóstico la mayoría de pacientes tiene CGD, en un análisis de 2280 pacientes del INEN el $66.8 \%$ de los pacientes ya tenía $\mathrm{M}^{(2)}$.

Los pacientes sin metástasis a distancia, o cáncer gástrico localizado (CGL), tienen opción de ser curados siempre y cuando se les realice una resección oncológica del tumor.

La resección oncológica ineludiblemente tiene que cumplir dos objetivos:

1.- Extirpación del tumor con márgenes libres.

2.- Resección de todos los ganglios regionales potencialmente comprometidos por la neoplasia.

\section{Márgenes libres}

Para obtener un margen libre de neoplasia, la distancia mínima entre el borde macroscópico del tumor depende del Tipo macroscópico del tumor (Precoz o Borrmann) y de la profundidad de invasión de la pared ${ }^{(3)}$ (Tabla 2).

Si luego de establecer el plano de sección (que garantice márgenes libres), queda aún un remanente gástrico funcional se realizará una gastrectomía sub total distal o proximal, en caso contrario se tendrá que resecar todo el estómago (Gastrectomía Total).

Si hay infiltración de estructuras vecinas (T4b) como colon, páncreas, hígado, etc., se debe efectuar una gastrectomía extendida combinada con resección en bloque de los órganos adyacentes infiltrados.

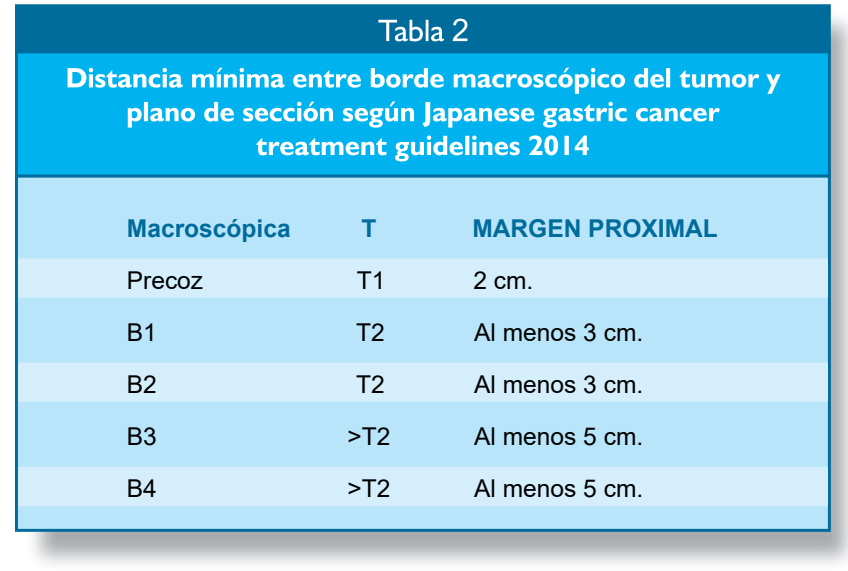

Si el tumor compromete el cardias, se debe realizar congelación del borde proximal de sección, un margen mayor a $5 \mathrm{~cm}$ no siempre es necesario.

\section{Resección grupos ganglionares (Linfadenectomía)}

La posibilidad de metástasis ganglionar regional guarda relación directa con la profundidad de invasión de la pared del estómago tal como se aprecia en la gráfica 1.

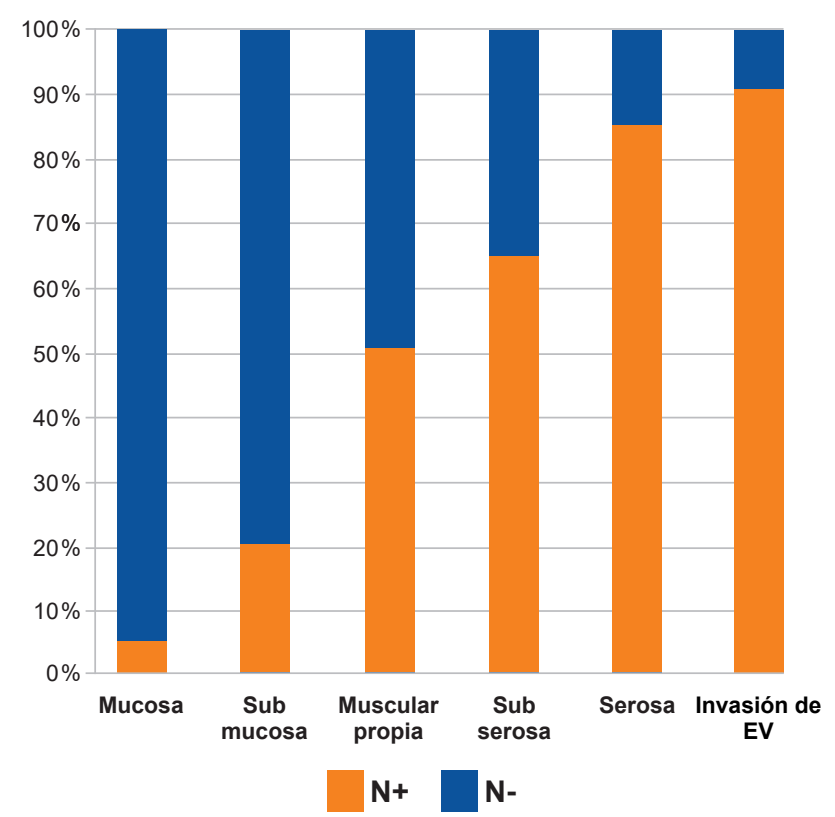

Gráfica 1. Porcentaje de metástasis ganglionar regional según profundidad de invasión de la pared gástrica.

A mayor $\mathrm{T}$ el porcentaje de $\mathrm{N}+$ se incrementa, por ejemplo la probabilidad de $\mathrm{N}+$ para un $\mathrm{T} 1 \mathrm{a}$ es de $5 \%$ pero para un T4b es de $90 \%$. 
Los ganglios regionales del estómago se catalogan en primera estación (Grupos 1-7) y segunda estación (Grupos 812), la resección de los ganglios (linfadenectomía) se clasifica en D0 si no se extrae ningún ganglio, D1 si se extirpa los ganglios de la primera estación y D2 si se resecan los ganglios de la primera y segunda estación.

Si bien los estudios clínicos controlados que comparan la sobrevida de D2 vs D1 no han encontrado diferencia en la sobrevida ${ }^{(4,5)}$, actualmente el manejo estándar es realizar una resección D2 y el número mínimo de ganglios regionales obtenido debe ser de al menos $16^{(1)}$.

Los grupos ganglionares que se resecan por tipo de linfadenectomía y gastrectomía se exponen en la tabla 3 (Japanese Gastric Cancer Association, 2017).

Debido a la heterogeneidad del CGL, el tipo de Gastrectomía (Total o sub total) y la extensión de la linfadenectomía (D0, D1 o D2) dependen de: localización del tumor (Antro, Cuerpo o Fondo), profundidad de invasión de la pared gástrica y de la posibilidad de metástasis ganglionar regional.

Por eso antes de decidir qué tipo de tratamiento requiere un CGL se deben usar todos los métodos diagnósticos posibles (Eco endoscopia, TAC, RMN) para precisar el estadio clínico TNM(cTNM).
De los múltiples algoritmos propuestos para el tratamiento quirúrgico, los publicados por la Japanese gastric cáncer guidelines ${ }^{(3)}$ son los más aceptados (Figuras 3-4).

Si en la estratificación pre operatoria se determina que el tumor es un T1a, menor de $2 \mathrm{~cm}$, no ulcerado y sin sospecha de metástasis ganglionar se indica una mucosectomía sin linfadenectomía (D0), pero si el tumor no cumple los criterios antes expuestos se amplía la linfadenectomía (D1, D1+), si hay sospecha de metástasis ganglionar la linfadenectomía siempre debe ser D2 ( Figura 3).

Para el cáncer gástrico avanzado (T2-T4, M0) la linfadenectomía siempre tiene que ser D2 tal como se aprecia en la figura 4.

\section{Resultados del tratamiento}

El Dr. Takeshi Sano ${ }^{(6)}$ realizó un estudio multicéntrico (59 instituciones), multinacional (15 países) para evaluar la sobrevida a 5 años de pacientes que tuvieron una resección oncológica por cáncer gástrico.

Los criterios de inclusión fueron:

-Adenocarcinoma histológicamente confirmado. del estómago o UEG (Siewert 2-3)

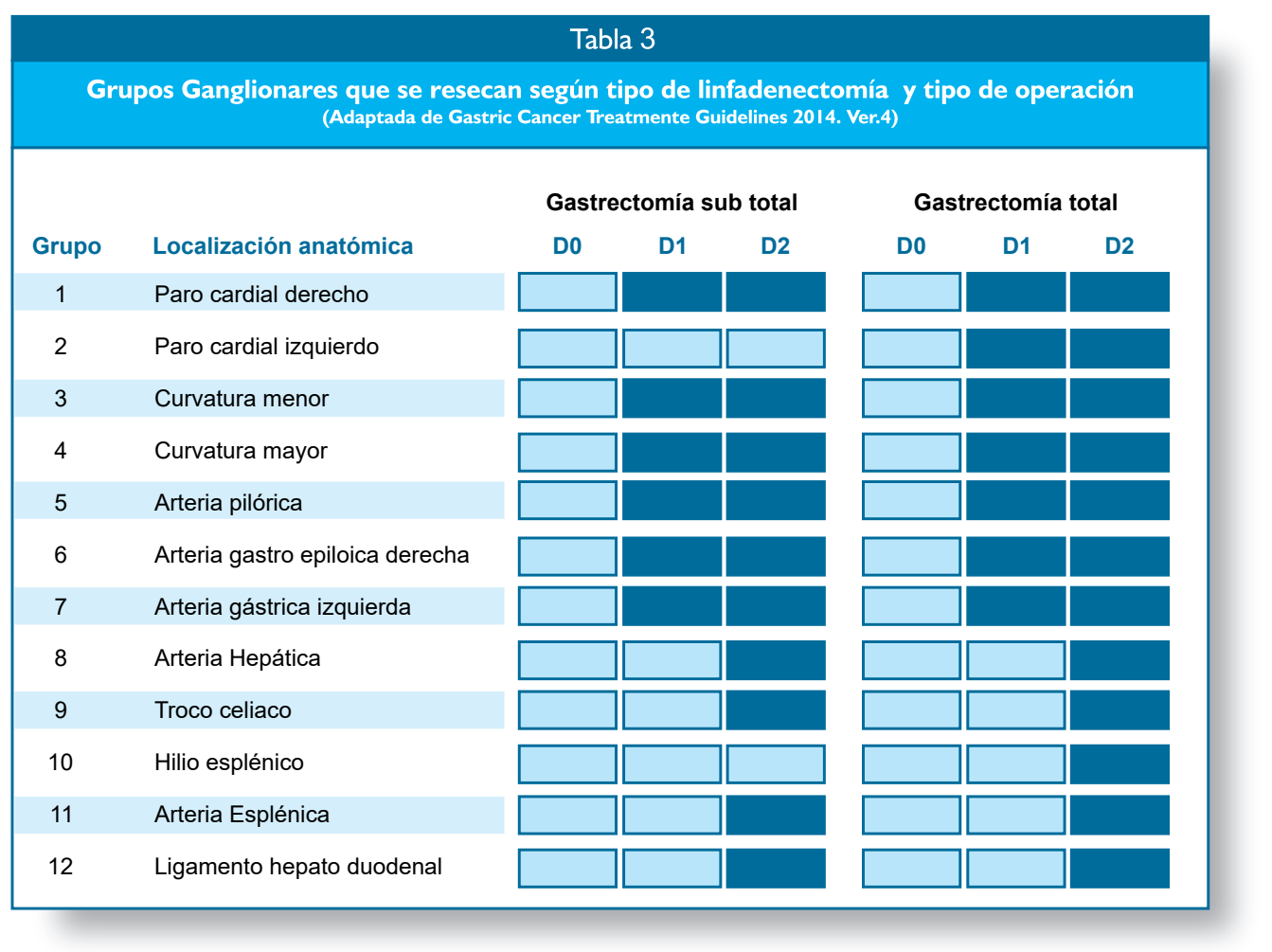




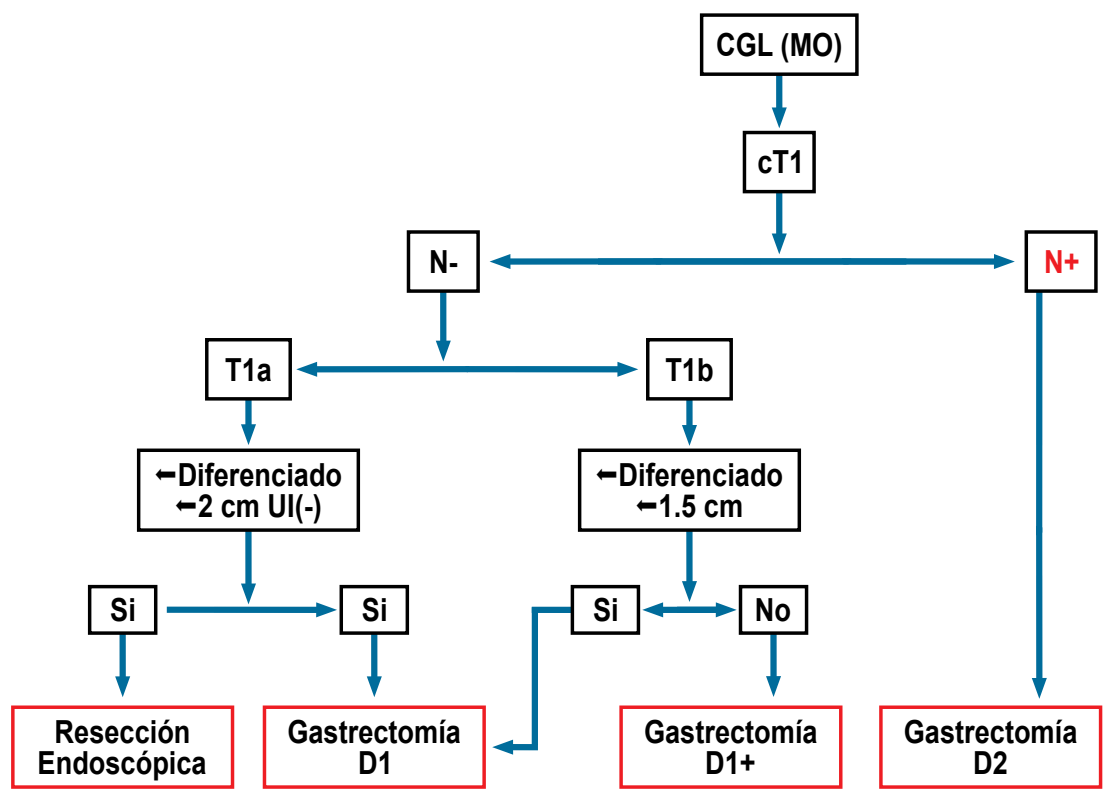

Figura 3. Tratamiento del Cáncer gástrico precoz según Japanese gastric cancer treatment guidelines 2014 (ver. 4).

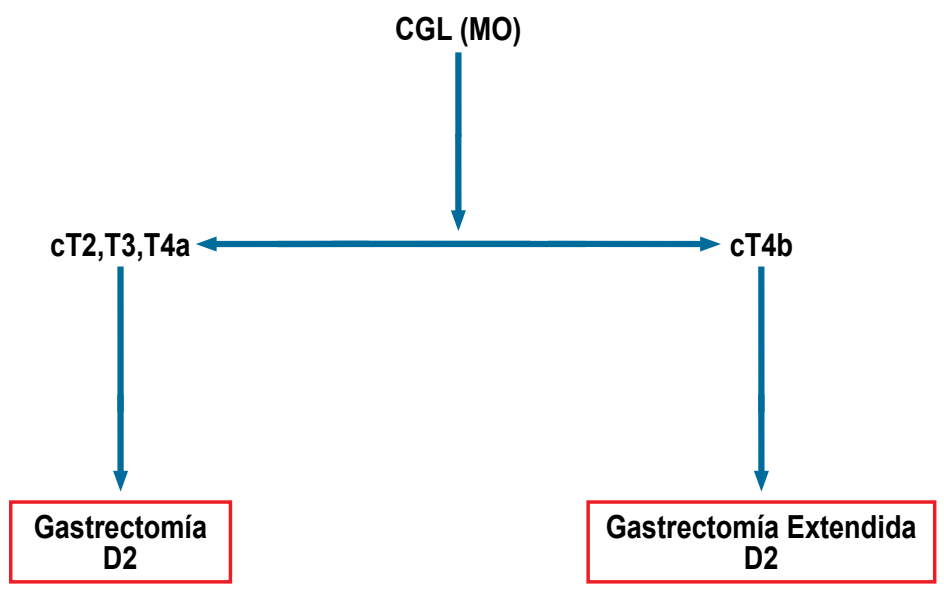

Figura 4. Tratamiento del Cáncer gástrico avanzado según Japanese gastric cancer treatment guidelines 2014 (ver. 4).

-Resección R0 realizada entre el 2000-2004

-Conocer si a los 5 años el paciente estaba vivo o muerto

-No Neo adyuvancia con Quimioterapia y/o Radioterapia.

-Los casos de cáncer del muñón y muertes operatorias fueron excluidos.

Al término del estudio se recolectó 25,411 pacientes, la gastrectomía distal fue la más frecuente en todas las regiones, seguida por la gastrectomía total y proximal, la linfadenectomía D2 fue realizada en la mayoría de los pacientes. El promedio de ganglios resecados fue 32 .

Las curvas de sobrevida a 5 años se muestran en la figura $5^{(6)}$.

En nuestra institución la resección oncológica del cáncer gástrico se estandarizó desde 1990, en la figura 6 se muestra la sobrevida a 10 años; nuestros resultados son similares a lo reportado por Sano ${ }^{(6)}$. 


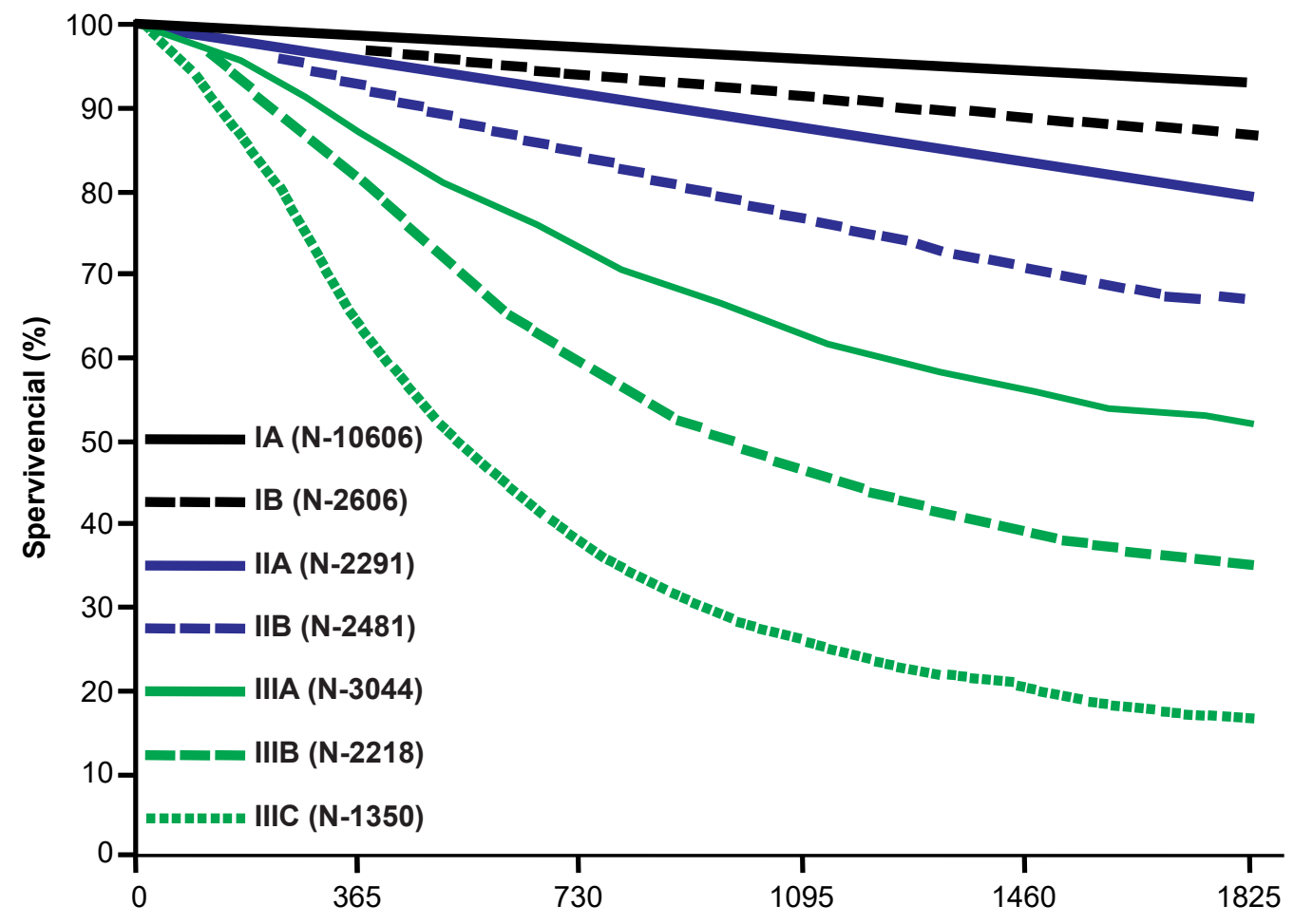

Figura 5. Sobrevida a 5 años según estadios PTNM Eight edition (Adaptado de Sano 2017)

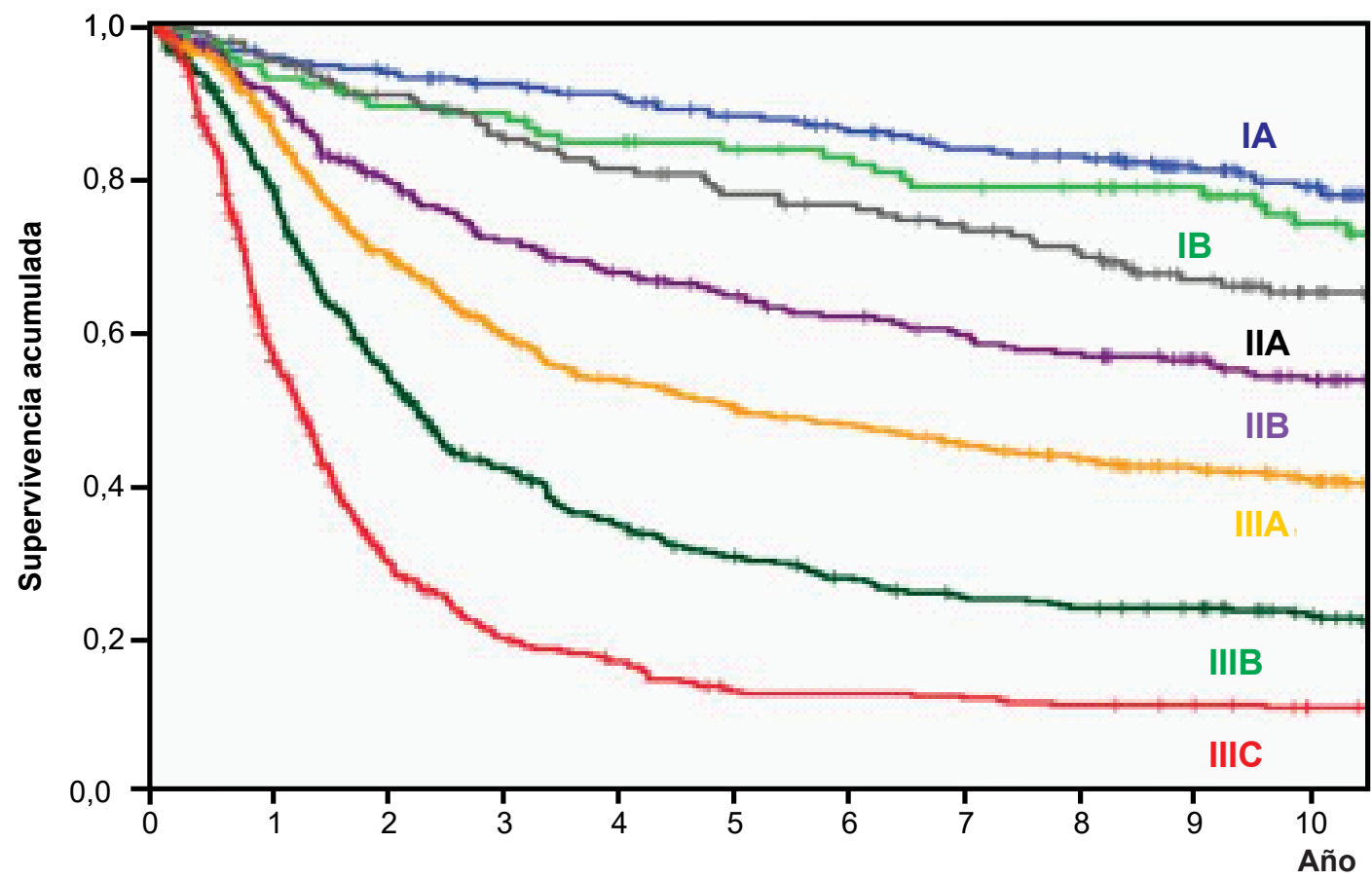

Figura 6. Sobrevida global a 10 años de pacientes con CGL que tuvieron una Gastrectomía D2 (INEN 2280 pacientes). 
Referencias bibliográficas

1.In H, Solsky I, Palis B, Langdon-Embry M, Ajani J, Sano T. Validation of the 8th Edition of the AJCC TNM Staging System for Gastric Cancer using the National Cancer Database. Annals of Surgical Oncology 2017;24(12):3683-3691. https://doi.org/ 10.12 45/s10434-017-6078-x.

2.Ruiz E, Berrospi F, Morante C, Payet E, Celis J, Montalbetti JA. Operability and resectability of gastric cancer: analysis of 2280 cases in 15 years. Rev Gastroenterologia Perú 1997;17(2): 135-142.

3.Japanese Gastric Cancer Association. Japanese gastric cancer reatment guidelines 2014. Gastric Cancer 2017;20(1):1-19. https://doi.org/10.1007/s10120-016-0622-4

4. Cuschieri A, Weeden S, Fielding J, Bancewicz J, Craven,J, Joypaul V, et al. Patient survival after D1 and D2 resections for gastric cancer: Long-term results of the MRC randomized surgical trial. Surgical Co-operative Group. British Journal of Cancer, 1999;79(9-10):1522-1530. https://doi.org/10.1038/ sj.bjc.6690 243.

5.Bonenkamp JJ, Hermans J, Sasako M, van de Velde CJ, Welvaart K, Songun I et al. \& Dutch Gastric Cancer Group. Extended lymph-node dissection for gastric cancer. N Engl J Med 1999;340(12):908-914. https://doi.org/10.1056/NEJM 199903253401202

6. Sano T, Coit DG, Kim HH, Roviello F, Kassab P, Wittekind C, et al. Proposal of a new stage grouping of gastric cancer for TNM classification: International Gastric Cancer Association staging project. Gastric Cancer: 2017;20(2):217-225. https://doi.org/ $10.1007 / \mathrm{s} 10120-016-0601-9$

Contribución de autoría: Eloy Ruiz ha participado en la concepción del artículo, la redacción del manuscrito,

la revisión crítica del manuscrito y aprobación de su versión final.

Conflicto de interés: Los autores no tienen conflictos de interés con la publicación de este trabajo.

Financiamiento: Autofinanciado.

Citar como: Ruiz, E. Tratamiento quirúrgico del cáncer gástrico. DIAGNÓSTICO (Lima). 2021;60(2):98-103.

DOI: 10.33734 /diagnostico.v60i2.287

Correspondencia: Eloy Ruiz. Correo electrónico: ruizeloy@gmail.com

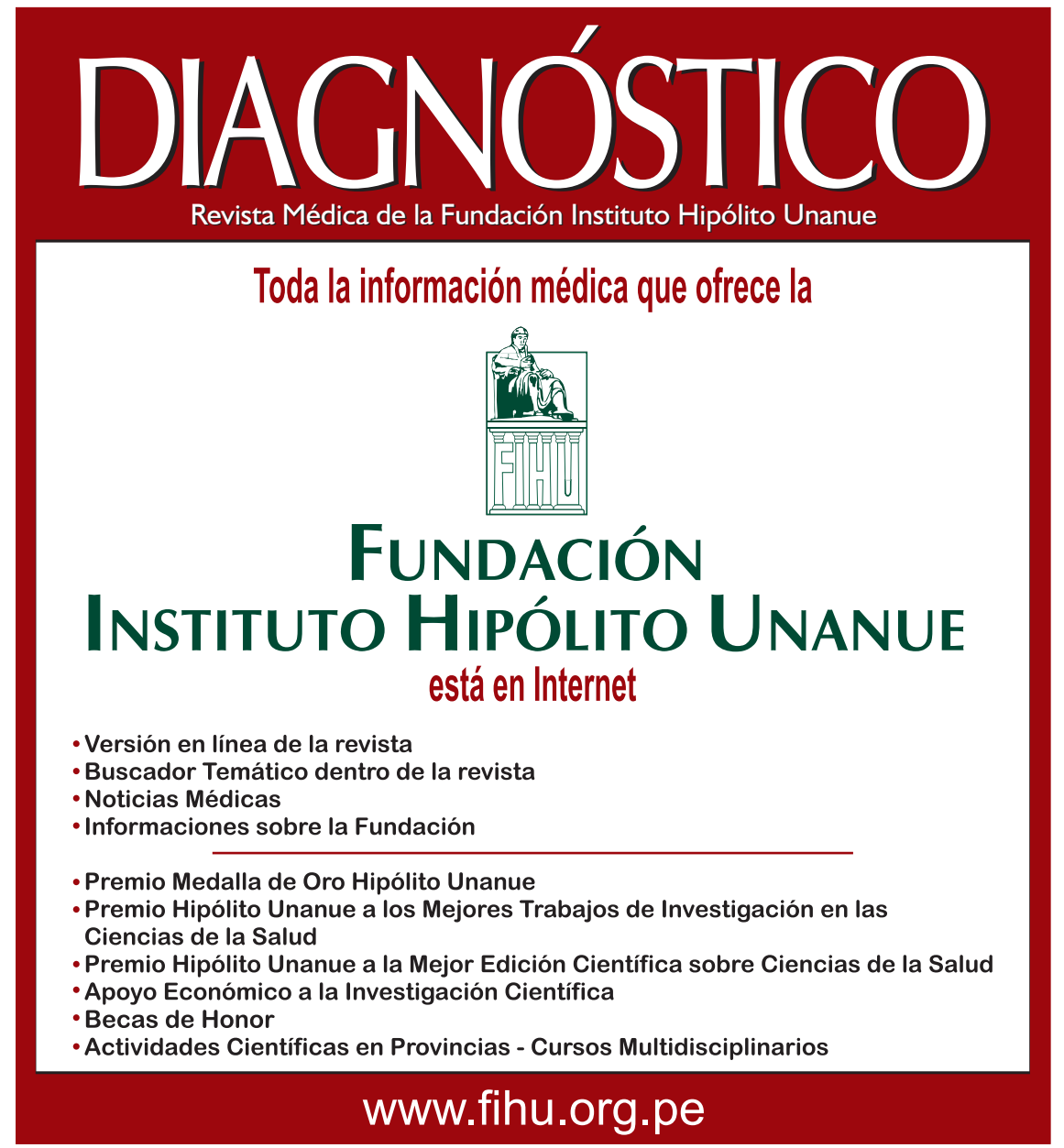

